\title{
Influência da formação acadêmica em Nutrição na expressão da neofobia alimentar
}

\author{
Influence of academic training in nutrition \\ on the incidence of neophobic feeding behavior
}

\author{
Diana Quitéria Cabral Ferreira ${ }^{1}$ \\ Felipe Nalon Castro ${ }^{2}$ \\ Fívia de Araújo Lopes ${ }^{2}$
}

${ }^{1}$ Escola da Saúde, Universidade Potiguar (UnP). Av. Senador Salgado Filho 1610, Lagoa Nova. 59056-000 Natal RN

Brasil.

dianaqcferreira@gmail.com

${ }^{2}$ Departamento de

Fisiologia, Centro de Biociências, Universidade Federal do Rio Grande do Norte. Natal RN Brasil.
Abstract This study sought to evaluate the influence of academic training in nutrition on the incidence of neophobic feeding behavior. It is a cross-sectional study conducted with 247 students, of both sexes and mean age of $24.6 \pm 6.8$ years, enrolled in the first and last year of the undergraduate course in nutrition and other higher education courses in the area of health science. The level of food neophobia was evaluated through the Food Neophobia Scale associated with a behavioral measure obtained from the choice between known or unknown food. In accordance with the results, the students who were embarking on the nutrition course had higher food neophobia scores than the students nearing the end of the course. However, this difference was not observed when compared with freshmen and seniors studying in other courses. In the behavioral assessment, it was seen that most of the seniors from the nutrition course chose the unknown preparation, while in the other groups the majority chose the known preparation. The results suggest that academic training in Nutrition may have an influence on the incidence of food neophobia.

Key words Feeding behavior, Students of health sciences, Food neophobia
Resumo O estudo objetivou avaliar a influência da formação acadêmica em Nutrição na expressão do comportamento alimentar neofóbico. Trata-se de um estudo transversal realizado com 247 universitários de ambos os sexos, com idade média de $24,6 \pm 6,8$ anos, matriculados no primeiro e no último ano de graduação em Nutrição e em outros cursos superiores da área de ciência da saúde. Foi avaliado o grau de neofobia alimentar através da Escala de Neofobia Alimentar associada a uma medida comportamental, obtida a partir da escotha entre alimentos conhecidos ou desconhecidos. De acordo com os resultados, os estudantes que estavam o início do curso de Nutrição apresentaram maiores escores de neofobia alimentar do que os do final do curso. No entanto, essa diferença não foi observada quando se comparou com os estudantes ingressantes e concluintes de outros cursos de graduação. Já na avaliação comportamental, foi visto que a maior parte dos estudantes concluintes do curso de Nutrição escolheu a preparação desconhecida, enquanto que nos demais grupos a maioria optou pela conhecida. Os resultados sugerem que a formação acadêmica em Nutrição pode ter influência sobre a expressão da neofobia alimentar.

Palavras-chave Comportamento alimentar, Estudantes de ciências da saúde, Neofobia alimentar 


\section{Introdução}

A composição de uma dieta adequada envolve uma série de fatores. Como onívoros, a inclusão de novos itens à dieta pode ser benéfica, mas pode também trazer consigo uma série de riscos, como, por exemplo, intoxicações alimentares ${ }^{1}$. Em sociedades caçadoras e coletoras, existe a constante necessidade de identificação e seleção dos itens alimentares. Por outro lado, em sociedades industrializadas, a maioria das pessoas tem acesso a informações importantes que auxiliam nas escolhas alimentares, como as nutricionais nos rótulos dos alimentos e características que indicam a qualidade dos mesmos, como o tipo de embalagem, data de fabricação, prazo de validade e análise das características organolépticas dos alimentos. Tais informações podem estar relacionadas à minimização dos riscos de consumir alimentos inapropriados ${ }^{2}$. Ainda assim, muitas pessoas evitam consumir novos alimentos ou experimentar preparações não familiares, ou seja, algumas pessoas podem apresentar um traço comportamental conhecido como neofobia alimentar.

A neofobia alimentar caracteriza-se pela relutância em consumir, ou falta de vontade em experimentar, alimentos desconhecidos ${ }^{3}$. $\mathrm{Na}$ perspectiva evolucionista, esse comportamento poderia ter apresentado vantagens ou desvantagens ao indivíduo, dependendo do ambiente em que o mesmo estivesse inserido. Por um lado, a neofobia alimentar poderia reduzir o risco de ingestão de alimentos maléficos à saúde, mas, em contrapartida, esse comportamento diminui a variedade de alimentos consumidos ${ }^{4-7}$.

Uma dieta restrita, em termos de variedade, pode desencadear vários danos à saúde humana, desde o estabelecimento de uma dieta monótona, o que propicia o aparecimento de carências nutricionais, até o desenvolvimento de morbidades crônicas relacionadas a uma alimentação desequilibrada, como o diabetes mellitus tipo II, doenças cardiovasculares e obesidade ${ }^{8,9}$. A frequência e o tipo de doenças dependem dos hábitos e do comportamento alimentar da população estudada ${ }^{9}$, dentre eles o neofóbico.

A expressão da neofobia alimentar pode ser modulada por diversos fatores, incluindo tanto os biológicos quanto os ambientais ${ }^{10,11}$. O conhecimento sobre as características nutricionais, a manipulação, o preparo e a exposição a diferentes alimentos ao longo da vida pode representar um importante papel na atenuação do comportamento neofóbico. Nesse sentido, o conhecimento adquirido ao longo do curso de graduação em Nutrição pode ser um fator que minimize a neofobia alimentar nesses estudantes, pois exige que o futuro nutricionista tenha propriedade das habilidades técnicas e profundo conhecimento sobre diversos aspectos da nutrição e dos alimentos. Ao adquirirem tais conhecimentos, espera-se que esses estudantes incorporem em seu comportamento hábitos alimentares saudáveis e nutricionalmente adequados, com uma dieta variada e equilibrada ${ }^{12}$.

No entanto, estudos relatam que transtornos alimentares e comportamento alimentar alterado são prevalentes em grupos específicos, como atletas, estudantes universitários, especialmente da área da saúde ${ }^{13-16}$, e profissionais que lidam com a corporeidade, dentre eles os nutricionistas e os estudantes do curso de Nutrição ${ }^{17,18}$.

Portanto, é de suma importância a investigação e a caracterização da neofobia alimentar entre estudantes de Nutrição, a fim de identificar o papel do conhecimento adquirido durante o curso sobre o comportamento alimentar neofóbico dos estudantes. Uma vez que serão nutricionistas que, no exercício da atenção dietética e planejamento dietoterápico, contribuirão ativamente no processo de mudança do comportamento alimentar dos indivíduos. Além disso, esses profissionais terão entre suas competências profissionais o dever de incluir conhecimentos sobre os alimentos e seus benefícios para a saúde no dia a dia da população, incentivar atitudes que busquem o estabelecimento de uma dieta variada e práticas alimentares saudáveis ${ }^{19}$. Até o momento, não existe na literatura o relato de pesquisas relacionando a influência da formação acadêmica sobre a expressão da neofobia alimentar.

Dessa forma, o presente trabalho buscou avaliar a influência da formação acadêmica em Nutrição na expressão do comportamento alimentar neofóbico entre estudantes universitários. A hipótese principal deste trabalho é que a formação acadêmica em Nutrição afeta a expressão do comportamento alimentar neofóbico. Para essa hipótese, tem-se como predições: a) Os estudantes que estão no final do curso de Nutrição apresentam menor neofobia alimentar que os do início do curso; b) Na avaliação do comportamento alimentar neofóbico, os estudantes concluintes do curso de Nutrição apresentarão preferência por alimentos novos. 


\section{Material e métodos}

\section{Delineamento da pesquisa}

Trata-se de um estudo de corte transversal desenvolvido com estudantes universitários no Laboratório de Nutrição Experimental e no Laboratório de Técnica Dietética e Tecnologia dos Alimentos da Universidade Potiguar (UnP), Natal, Rio Grande do Norte, Brasil. A coleta de dados ocorreu entre abril e junho de 2012, nos turnos matutino, vespertino e noturno. O protocolo da pesquisa foi aprovado pelo Comitê de Ética em Pesquisa da Universidade Federal do Rio Grande do Norte (UFRN).

\section{Participantes}

A amostragem foi feita de forma probabilística e sem reposição. O tamanho da amostra foi identificado com auxílio de cálculo de poder estatístico, utilizando o software $\mathrm{G}^{*}$ Power 3.1.9.2 ${ }^{20}$, assumindo $\alpha=0,05$, Poder $=0,80$ e medida de efeito (f) $=0,28$.

Participaram da pesquisa 247 estudantes universitários devidamente matriculados nos cursos da referida instituição de ensino superior no ano de 2012, adultos e de ambos os sexos. Os participantes foram agrupados de acordo com o período, primeiro (ingressantes) ou último ano (concluintes), e curso de graduação ao qual estavam vinculados, Nutrição ou outros cursos da área da saúde, incluindo os cursos de Ciências Biológicas, Educação Física, Enfermagem, Farmácia, Fisioterapia, Fonoaudiologia, Odontologia, Medicina, Psicologia e Serviço Social. Resultou-se em quatro grupos: Nutrição - ingressante ( $\mathrm{n}=$ 121); Nutrição - concluinte $(\mathrm{n}=44)$; Outros cursos superiores da área da saúde - ingressante $(\mathrm{n}=$ 39); e Outros cursos superiores da área da saúde - concluinte $(\mathrm{n}=43)$. Não foram incluídos os estudantes dos períodos intermediários dos cursos estudados, bem como aqueles que relataram intolerância ou restrições alimentares com relação aos alimentos utilizados na pesquisa.

\section{Procedimentos}

Inicialmente os participantes foram recrutados nas suas respectivas salas de aula e convidados a participarem da pesquisa. Todos foram esclarecidos quanto aos objetivos e procedimentos da pesquisa e aqueles que aceitaram participar assinaram o Termo de Consentimento Livre e Esclarecido (TCLE). Em seguida, com o intuito de melhor caracterizar a amostra estudada, os participantes responderam um questionário autopreenchível contendo questões sociodemográficas, como sexo, idade, cor da pele, indicada segundo a Classificação Brasileira de cor de pele ${ }^{21}$, estado civil e nível socioeconômico, que foi avaliado pelo instrumento da Associação Brasileira de Empresas de Pesquisa ${ }^{22}$, tendo como base os bens existentes no domicílio (eletrodomésticos e carros), presença de empregada doméstica e escolaridade do chefe da família. O escore obtido por cada participante foi tratado como variável categórica, variando de A (nível mais elevado) até $\mathrm{E}$ (nível mais baixo). Posteriormente os estudantes fizeram um ensaio comportamental com relação à neofobia alimentar, escolhendo entre a preparação conhecida ou a desconhecida, e logo após responderam a Escala de Neofobia Alimentar, conforme descrito a seguir.

\section{Escala de Neofobia Alimentar}

Os participantes responderam uma versão brasileira da Escala de Neofobia Alimentar $(\mathrm{ENA})^{3}$, que aborda atitudes relacionadas aos alimentos desconhecidos e ao comportamento alimentar. Essa escala é composta por 10 itens, os quais são apresentados na forma de afirmações avaliadas em escala de Likert de sete pontos, variando de "discordo totalmente" (1) a "concordo totalmente" (7). Por meio desse instrumento os participantes devem indicar o quanto concordam com as afirmações sobre aceitação e desconfiança com relação a alimentos novos e diferentes, preferência por alimentos estrangeiros e se apresenta facilidade em consumir diversos tipos de alimentos, inclusive os desconhecidos.

O processo de tradução da ENA para a língua portuguesa foi realizado por indivíduos fluentes em ambos idiomas (português e inglês). As dez afirmações foram traduzidas por três tradutores bilíngues, de forma independente. Tais versões foram traduzidas para o inglês (retrotradução) por outros três indivíduos bilíngues. A adaptação do questionário para a língua portuguesa foi realizada utilizando a técnica back-translation. As versões traduzidas foram comparadas com a versão original e realizados os ajustes necessários, a fim de se obter a equivalência conceitual e linguística, chegando, assim, à versão final da escala em português utilizada na pesquisa.

O cálculo para determinação do escore de neofobia alimentar foi realizado conforme descrito pelos autores da escala original ${ }^{3}$. O escore da ENA foi obtido a partir da soma das respos- 
tas, após reverter os escores das questões 1, 4, 6 e 9, com pontuação final variando entre 10 e 70 pontos.

\section{Comportamento Alimentar Neofóbico}

$\mathrm{Na}$ avaliação da medida comportamental com relação à neofobia alimentar, foi observada a atitude perante a escolha de alimentos-estímulo, visando verificar se os escores apresentados na ENA correspondiam ao comportamento dos participantes. Nessa avaliação foram utilizados como alimentos estímulo patês elaborados com alimentos possivelmente conhecidos e familiares aos participantes, como a azeitona e presunto, e com alimentos possivelmente novos e desconhecidos, como alcachofra e anchova. A identificação quanto ao grau de conhecimento com relação aos alimentos-estímulo apresentados foi feita pelo próprio participante no momento da atividade.

Nessa etapa os participantes foram alocados em cabines individuais e expostos a dois tipos de patês, sendo um deles possivelmente conhecido e o outro possivelmente desconhecido, juntamente com torradas. Os estudantes foram questionados individualmente quanto à familiaridade e preferência com relação aos alimentos apresentados e, em seguida, foram convidados a selecionarem e a degustarem um dos dois patês apresentados.

\section{Análise estatística}

Os pressupostos de normalidade da distribuição das variáveis e da homogeneidade das variâncias para as médias dos grupos comparados foram avaliados usando o teste de Kolmogorov -Smirnov e Levene, respectivamente. O Modelo Linear Geral (GLM) 2x2 foi aplicado para avaliar os fatores que influenciam na neofobia alimentar. Nesse teste foi utilizada como variável dependente a pontuação da ENA e como variáveis independentes o curso superior dos estudantes (Nutrição e outros cursos) e o período que estavam cursando (ingressantes e concluintes). Nas análises post-hoc foi utilizado o teste $t$ de Student, utilizando a correção de Bonferroni para o nível de significância. Para observar a associação entre a resposta comportamental (escolha entre o alimento familiar ou novo) e os grupos estudados foi utilizado o teste do Qui-quadrado, utilizando o resíduo ajustado, em que valores maiores que 1,96 indicam que há evidências de associação significativa entre as categorias analisadas. $\mathrm{O}$ nível de significância adotado nas análises foi 5\%.

\section{Resultados}

As características sociodemográficas dos estudantes que participaram da pesquisa estão apresentadas na Tabela 1. A média de idade encontrada foi $24,6 \pm 6,8$ anos, variando entre 19 e 51 anos, estando a maior parte da amostra na faixa etária entre 19 e 25 anos. A maioria dos participantes era do sexo feminino, em todos os grupos estudados, e referiu possuir cor de pele parda. Com relação ao estado civil, a maioria afirmou estar em um relacionamento (namorando ou casado). E quanto a classificação econômica, a maior parte pertencia as classes $\mathrm{B}$ e C.

Foi identificada uma interação entre o curso que o estudante estava cursando e o perídio de formação $(F(1,243)=4,89, p=0,028)$. De maneira geral, a expressão da neofobia alimentar, avaliada pela ENA, foi diferente entre os estudantes do curso de Nutrição e de outros da área da saúde nos dois períodos avaliados. Não foi encontrado efeito isolado do curso $(F(1,243)=1,01, p=$ $0,317)$ e do período, se ingressante ou concluinte $(F(1,243)=0,72, p=0,396)$.

As análises post-hoc mostraram que os estudantes do curso de Nutrição que estavam no primeiro ano do curso apresentaram valores mais elevados na ENA $(38,8 \pm 11,0$ pontos $)$, quando comparados aos concluintes ( $34,5 \pm 10,5$ pontos) $(t(163)=2,42, p=0,017)$. Não foi observada diferença estatisticamente significativa entre os valores obtidos na ENA entre os estudantes ingressantes e os concluintes dos outros cursos superiores da área da saúde $(t(80)=-0,90, p=0,370)$. Embora esse último resultado não tenha sido estatisticamente significativo, há uma tendência de aumento na expressão da neofobia alimentar nos estudantes concluintes $(39,0 \pm 10,4$ pontos), quando comparados com os ingressantes dos outros cursos da área da saúde $(37,1 \pm 8,8$ pontos), como pode ser observado na Figura 1.

O resultado originado da análise do Qui-quadrado indicou associação entre a medida comportamental e o curso/período dos estudantes $\left(\chi^{2}(2)=8,74, p=0,033\right)$, ou seja, a maior parte dos estudantes concluintes do curso de Nutrição escolheu a preparação desconhecida, enquanto que nos demais grupos os resultados foram muito próximos do esperado (Tabela 2).

\section{Discussão}

O comportamento alimentar é complexo e inclui determinantes internos e externos ao indivíduo. 
Tabela 1. Dados sociodemográficos dos participantes da pesquisa. Rio Grande do Norte, 2012.

\begin{tabular}{|c|c|c|c|c|c|c|c|c|}
\hline & \multicolumn{2}{|c|}{$\begin{array}{l}\text { Nutrição- } \\
\text { Ingressantes } \\
(\mathrm{n}=121)\end{array}$} & \multicolumn{2}{|c|}{$\begin{array}{l}\text { Nutrição - } \\
\text { Concluintes } \\
(\mathrm{n}=44)\end{array}$} & \multicolumn{2}{|c|}{$\begin{array}{l}\text { Outros cursos - } \\
\text { Ingressantes } \\
\quad(\mathbf{n}=39)\end{array}$} & \multicolumn{2}{|c|}{$\begin{array}{l}\text { Outros cursos - } \\
\text { Concluintes } \\
(n=43)\end{array}$} \\
\hline & $\mathbf{n}$ & $\%$ & $\mathbf{n}$ & $\%$ & $\mathbf{n}$ & $\%$ & n & $\%$ \\
\hline \multicolumn{9}{|l|}{ Faixa etária } \\
\hline$\leq 25$ anos & 93 & 76,9 & 17 & 38,6 & 32 & 82,1 & 31 & 72,1 \\
\hline$>25$ anos & 28 & 23,1 & 27 & 61,4 & 07 & 17,9 & 12 & 27,9 \\
\hline \multicolumn{9}{|l|}{ Sexo } \\
\hline Feminino & 112 & 92,6 & 40 & 90,9 & 30 & 69,8 & 30 & 85,8 \\
\hline Masculino & 09 & 7,4 & 04 & 9,1 & 09 & 30,2 & 13 & 14,2 \\
\hline \multicolumn{9}{|l|}{ Cor da pele } \\
\hline Branca & 50 & 41,3 & 18 & 40,9 & 16 & 41,0 & 14 & 32,6 \\
\hline Parda & 51 & 42,1 & 19 & 43,2 & 17 & 43,6 & 19 & 44,2 \\
\hline Outras & 20 & 16,6 & 07 & 15,9 & 06 & 15,4 & 10 & 23,3 \\
\hline \multicolumn{9}{|l|}{ Estado civil } \\
\hline Solteiro & 71 & 58,7 & 21 & 47,7 & 26 & 66,7 & 24 & 55,8 \\
\hline Casado & 24 & 19,8 & 15 & 34,1 & 05 & 12,8 & 08 & 18,6 \\
\hline Outros & 26 & 21,5 & 08 & 18,2 & 08 & 20,5 & 11 & 25,6 \\
\hline \multicolumn{9}{|c|}{ Classe econômica } \\
\hline A & 03 & 2,5 & 04 & 9,1 & 0 & 0,0 & 0 & 0,0 \\
\hline B & 44 & 36,4 & 23 & 52,3 & 19 & 48,7 & 21 & 48,8 \\
\hline $\mathrm{C}$ & 66 & 54,5 & 16 & 36,3 & 18 & 46,2 & 18 & 41,9 \\
\hline $\mathrm{D}$ e $\mathrm{E}$ & 08 & 6,6 & 01 & 2,3 & 02 & 5,1 & 04 & 9,3 \\
\hline
\end{tabular}

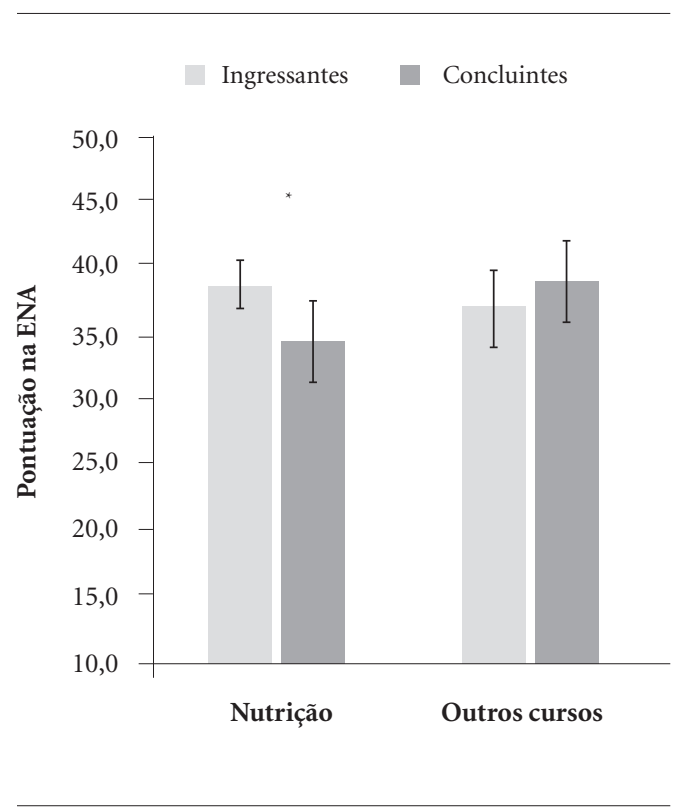

Figura 1. Médias e intervalos de confiança da pontuação na Escala de Neofobia Alimentar (ENA) dos estudantes, ingressantes e concluintes, do curso de Nutrição e de outros cursos superiores da área da saúde. Rio Grande do Norte, 2012. ${ }^{*} \mathrm{p}=0,017$.
Os fatores externos, como por exemplo, o contexto social onde o indivíduo está inserido, a renda, a cultura e o estilo alimentar, são os mais estudados na avaliação do comportamento alimentar, especialmente quando se propõem estratégias de mudança dos hábitos alimentares para promoção da saúde. A neofobia alimentar apresenta importante papel na formação do hábito alimentar e vem sendo amplamente estudada em indivíduos adultos ${ }^{5,23}$, principalmente devido à sua influência na variedade de alimentos consumidos ${ }^{24,25}$ e de seu possível efeito na adesão a planejamentos dietoterápicos ${ }^{26,27}$. No estudo de Quick et al. ${ }^{28}$ envolvendo pacientes com diabetes mellitus tipo I foi observado que a neofobia alimentar estava negativamente associada à variedade da dieta dos participantes e também à adesão ao tratamento.

A amostra avaliada neste estudo foi predominantemente do sexo feminino, especialmente no curso de Nutrição, o que pode representar um fator importante nos achados do presente trabalho, pois estudos prévios com estudantes universitários demonstraram que as mulheres apresentam maiores escores de neofobia alimentar ${ }^{5}$.

Estudos demostraram que a exposição repetida a novos alimentos ou preparações possui efei- 
Tabela 2. Associação entre a resposta do ensaio comportamental para a neofobia alimentar e o curso e período dos participantes. Rio Grande do Norte, 2012.

\begin{tabular}{llrrr}
\hline & & \multicolumn{2}{c}{ Escolha do Alimento } & \multirow{2}{*}{ Total } \\
\cline { 3 - 4 } Nutrição - Ingressantes & & Familiar & Novo & \\
& Observado & 74,0 & 47,0 & \multirow{2}{*}{121,0} \\
& Esperado & 67,1 & 53,9 & 121,0 \\
Nutrição - Concluintes & Resíduo & 1,8 & $-1,8$ & \\
& Observado & 16,0 & 28,0 & 44,0 \\
Outros cursos - Ingressantes & Esperado & 24,4 & 19,6 & 44,0 \\
& Resíduo & $-2,8$ & 2,8 & \\
Outros cursos - Concluintes & Observado & 24,0 & 15,0 & 39,0 \\
& Esperado & 21,6 & 17,4 & 39,0 \\
& Resíduo & 0,8 & $-0,8$ & \\
& Observado & 23,0 & 20,0 & 43,0 \\
& Esperado & 23,9 & 19,1 & 43,0 \\
& Resíduo & $-0,3$ & 0,3 & \\
\hline
\end{tabular}

Nota. $\chi 2(3)=8,74, p=0,033$. Os valores em negrito indicam a escolha do alimento com valores acima do esperado para os respectivos grupos.

to importante sobre a neofobia alimentar ${ }^{29}$. Mas, além disso, também foi evidenciado que o comportamento alimentar neofóbico pode ser amenizado quando se tem acesso a informações sobre esses alimentos desconhecidos ${ }^{30,31}$. Os escores de neofobia alimentar observados na amostra pesquisada foram semelhantes aos encontrados em outras pesquisas que também utilizaram a ENA em adultos ${ }^{3,23,30}$. Os resultados desta pesquisa sugerem que o conhecimento que os estudantes de Nutrição adquirem durante seu curso de graduação pode influenciar a expressão da neofobia alimentar, avaliada com base na ENA e no ensaio comportamental, sendo observada de maneira geral menor neofobia nos estudantes do último ano do curso de Nutrição.

Diversos estudos mostram que os estudantes apresentam mudanças significativas no seu hábito alimentar após a entrada na universidade, elevando o consumo de alimentos industrializados e rápidos de serem preparados e consumidos, o que pode se dever ao acesso limitado de alimentos saudáveis, nível de estresse elevado relacionado aos novos desafios enfrentados, ou mesmo aos maus hábitos alimentares pré-estabelecidos ${ }^{32-34}$. Na pesquisa desenvolvida por Matias e Fiore ${ }^{35}$ com discentes de Nutrição foi observado que a maior parte dos estudantes do último ano do curso referiu ter melhorado os hábitos alimentares e atribuiu tal mudança ao conhecimento adquirido duran- te a essa formação acadêmica. Nesse sentido, pela própria noção de que mudanças no comportamento alimentar acontecem de forma gradual e, na maioria das vezes, demorada, podendo variar entre as pessoas, Brown et al..$^{36}$ sugerem o desenvolvimento e a implementação de ações contínuas de educação alimentar e nutricional para alunos de Nutrição desde o seu ingresso ao curso.

Em se tratando de futuros nutricionistas, fica clara a importância de uma abordagem ampla na formação desses profissionais sobre a composição do comportamento alimentar e os fatores que o podem influenciar. Acreditar de forma crítica no conhecimento adquirido é fundamental para agir no comportamento alimentar de outras pessoas $^{37}$. O nutricionista possui o papel de educador, devendo auxiliar indivíduos a estabelecer práticas e hábitos alimentares adequados às suas necessidades nutricionais, considerando os recursos alimentares locais e o padrão cultural ${ }^{19,37}$, evitando ao máximo que suas preferências e aversões alimentares pessoais intervenham negativamente no planejamento dietético proposto.

Considerando todo o exposto, uma questão importante que pode ser levantada, embora não tenha sido explorada neste estudo, se refere ao questionamento do quanto essas características do comportamento alimentar individual são capazes de impactar na atitude do indivíduo frente à atividade profissional. Dentre as limita- 
ções deste estudo está o delineamento transversal adotado, ou seja, a estimativa das relações entre as variáveis em apenas um único momento deixa margem para o aprofundamento da relação entre o conhecimento adquirido durante o curso e a expressão do comportamento alimentar neofóbico. Outra limitação do estudo é o uso de uma versão da Escala de Neofobia Alimentar ainda não validada para a língua portuguesa.

É importante destacar que, dentro do conhecimento dos autores, este é o primeiro estudo brasileiro que investigou a neofobia alimentar em estudantes de Nutrição, comparando dois momentos distintos no decorrer da formação acadêmica: o inicial e o final. Sendo observado que a formação acadêmica em Nutrição e o conhecimento adquirido ao longo do curso parecem ter influência sobre a expressão da neofobia alimentar. Estudos como este podem contribuir para a orientação de estratégias de formação profissional que visem à identificação e à diminuição de comportamentos de risco que possam afetar negativamente as condutas profissionais dos futuros nutricionistas.

\section{Colaboradores}

DQC Ferreira participou da coleta, análise e discussão dos resultados, bem como da redação do artigo. FN Castro colaborou com a análise estatística dos dados, relato e discussão dos resultados e na redação final do artigo. FA Lopes coordenou toda a pesquisa, participou em todos as etapas do estudo e colaborou na redação final do artigo.

\section{Agradecimentos}

Gostaríamos de agradecer a equipe que auxiliou na coleta dos dados: Amanda Valeska Araújo Silva, Camila Cortez de Lima, Ivanise Augusta Luna Sandes, Janaiza Carla de Oliveira Lima, Luzia Elionaide A. Martins e Raí Nabichedí da Silva. Também somos gratos a Universidade Potiguar pelo suporte financeiro e disponibilização do espaço para realização da pesquisa. Por fim, mas não menos importante, agradecemos imensamente aos estudantes, que gentilmente aceitaram participar da pesquisa.

\section{Referências}

1. Rozin P. Sociocultural influences on human food selection. In: Capaldi ED, organizadora. Why we eat what we eat. Washington, DC: American Psychological Association; 1996. p. 233-262.

2. Teixeira PDS, Reis BZ, Vieira DAS, Costa D, Costa JO, Raposo OFF, Wartha ER, Netto RS. Educational nutritional intervention as an effective tool for changing eating habits and body weight among those who practice physical activities. Cien Saude Colet 2013; 18(2):347356.

3. Pliner P, Hobden K. Development of a scale to measure the trait of food neophobia in humans. Appetite 1992; 19(2):105-120

4. Falciglia GA, Couch SC, Gribble LS, Pabst SM, Frank R. Food neophobia in childhood affects dietary variety. $J$ Am Diet Assoc 2000; 100(1):1474-1478.

5. Lopes FA, Cabral JSP, Spinelli LHP, Cervenka L, Yamamoto ME, Branco RC, Hattori WT. Comer ou não comer, eis a questão: diferenças de gênero na neofobia alimentar. Psico-USF 2006; 11(1):123-125.

6. Yamamoto ME, Lopes FA. A Evolução do comportamento alimentar: selecionando o que comer. Rev FAPERN 2006; 1(4):21-23.

7. Knaapila A, Tuorila H, Silventoinen K, Keskitalo K, Kallela M, Wessman M, Peltonen L, Cherkas LF, Spector TD, Perola M. Food neophobia shows heritable variation in humans. Physiol Behav 2007; 91(5):573-578.

8. Skouteris H, McCabe M, Swinburn B, Hill B. Healthy eating and obesity prevention for preschoolers: a randomised controlled trial. BMC Public Health 2010; 10:220. 
9. Jew S, AbuMweis SS, Jones PJH. Evolution of the human diet: linking our ancestral diet to modern functional food as a means of chronic disease prevention. J Med Food 2009; 12(5):925-934.

10. Knaapila A, Silventoinen K, Broms U, Rose RJ, Perola M, Kaprio J, Tuorila HM. Food neophobia in young adults: genetic architecture and relation to personality, pleasantness and use frequency of foods, and body mass index-a twin study. Behav Genet 2011; 41(4):512521.

11. Blissett J, Fogel A. Intrinsic and extrinsic influences on children's acceptance of new foods. Physiology \& Behavior 2013; 121:89-95.

12. Penz LR, Dal Bosco SM, Vieira JM. Risco para desenvolvimento de transtornos alimentares em estudantes de Nutrição. Sci Med 2008; 18(3):124-128.

13. Liao Y, Knoesen NP, Castle DJ, Tang J, Deng Y, Bookun R, Chen X, Hao W, Meng G, Liu T. Symptoms of disordered eating, body shape, and mood concerns in male and female Chinese medical students. Compr Psychiatry 2010; 51(5):516-523.

14. Alvarenga MS, Lourenço BH, Philippi ST, Scagliusi FB. Disordered eating among Brazilian female college students. Cad Saude Publica 2013; 29(5):879-888.

15. Laus MF, Moreira RCM, Costa TMB. Diferenças na percepção da imagem corporal, no comportamento alimentar e no estado nutricional de universitárias das áreas de saúde e humanas. Rev Psiquiatr 2009; 31(3):192-196

16. Alves HJ, Boog MCF. Comportamento alimentar em moradia estudantil: um espaço para promoção da saúde. Rev Saude Publica 2007; 41(2):197-204.

17. Magalhães P, Motta DG. Uma abordagem psicossocial do estado nutricional e do comportamento alimentar de estudantes de nutrição. Nutrire: Rev Soc Bras Alim Nutr 2012; 37(2):118-132.

18. Silva JD, Silva ABJ, Oliveira AVK, Nemer ASA. Influência do estado nutricional no risco para transtornos alimentares em estudantes de nutrição. Cien Saude Colet 2012; 17(12):3399-3406.

19. Conselho Federal de Nutricionistas (CFN). Resolução $n^{\circ} 380 / 2005$. Dispõe sobre a definição das áreas de atuação do nutricionista e suas atribuições, estabelecem parâmetros numéricos de referência, por área de atuação, e dá outras providências. Diário Oficial da União 2006; 10 jan.

20. Faul F, Erdfelder E, Lang AG, Buchner A. G*Power 3: A flexible statistical power analysis program for the social, behavioral, and biomedical sciences. Behavior Research Methods 2007; 39(2):175-191.

21. Instituto Brasileiro de Geografia e Estatística (IBGE). Sintese de indicadores sociais: Uma análise das condições de vida da população Brasileira. Rio de Janeiro: IBGE; 2010. Estudos \& pesquisas: Informação demográfica e socioeconômica (número 27)

22. Associação Brasileira de Empresas de Pesquisa (ABEP). Critério de Classificação Econômica Brasil. Dados com base no Levantamento Socioeconômico. Rio de Janeiro: ABEP; 2012.

23. Olabi A, Najm NEO, Baghdadi OK, Morton JM. Food neophobia levels of Lebanese and American college students. Food Qual Prefer 2009; 20(5):353-362.
24. Mustonen S, Oerlemans P, Tuorila $\mathrm{H}$. Why don't they like that? And can I do anything about it? The nature and correlates of parents' attributions and self-efficacy beliefs about preschool children's food preferences. Appetite 2013; 66:34-43.

25. Siegrist M, Hartmann C, Keller C. Antecedents of food neophobia and its association with eating behavior and food choices. Food Qual Prefer 2013; 30(2):293-298.

26. Svensson V, Lundborg L, Cao Y, Nowicka P, Marcus C, Sobko T. Obesity related eating behaviour patterns in Swedish preschool children and association with age, gender, relative weight and parental weight - factorial validation of the Children's Eating Behaviour Questionnaire. Int J Behav Nutr Phys Act 2011; 8:134.

27. Skouteris H, McCabe M, Swinburn B, Hill B. Study protocol Healthy eating and obesity prevention for preschoolers: a randomised controlled trial. BMC Public Health 2010; 10:220.

28. Quick V, Lipsky LM, Laffel LMB, Mehta SN, Quinn H, Nansel TR. Relationships of neophobia and pickiness with dietary variety, dietary quality and diabetes management adherence in youth with type 1 diabetes. Eur $J$ Clin Nutr 2014; 68(1):131-136.

29. Ventura AK, Worobey J. Early Influences on the Development of Food Preferences. Curr Biol 2013; 23(9):401-408.

30. Dovey TM, Aldridge VK, Dignan W, Staples PA, Gibson EL, Halford JCG. Developmental differences in sensory decision making involved in deciding to try a novel fruit. Brit J Health Psychol 2012; 17(2):258-272.

31. Seo S, Kim OY, Oh S, Yun N. Influence of informational and experiential familiarity on image of local foods. Int J Hosp Manag 2013; 34:295-308.

32. Driskell JA, Schake MC, Detter HA. Using nutrition labeling as a potential tool for changing eating habits of university dining hall patrons. J Am Diet Assoc 2008; 108(12):2071-2076.

33. Kelly NR, Mazzeo SE, Bean MK. Systematic Review of Dietary Interventions With College Students: Directions for Future Research and Practice. J Nutr Educ Behav 2013; 45(4):304-313.

34. Strong KA, Parks SL, Anderson E, Winett R, Davy BM. Weight gain prevention: identifying theory-based targets for health behavior change in young adults. $J \mathrm{Am}$ Diet Assoc 2008; 108(10):1708-1715.

35. Matias CT, Fiore EG. Mudanças no comportamento alimentar de estudantes do curso de nutrição em uma instituição particular de ensino superior. Nutrire: Rev Soc Bras Alim Nutr 2010; 35(2):53-66.

36. Brown K, Wengreen H, Dimmick M, Eller K, Frampton A, Heaton E, Christensen N. Improving diets of college students: survey of dietary habits and focus group perspectives on how to best teach students. J Health Behav \& Pub Health 2011; 1(1):23-29.

37. Viana V. Psicologia, saúde e nutrição: Contributo para o estudo do comportamento alimentar. Anál Psicol 2002; 20(4):611-624.

Artigo apresentado em 14/04/2015

Aprovado em 22/10/2015

Versão final apresentada em 24/10/2015 